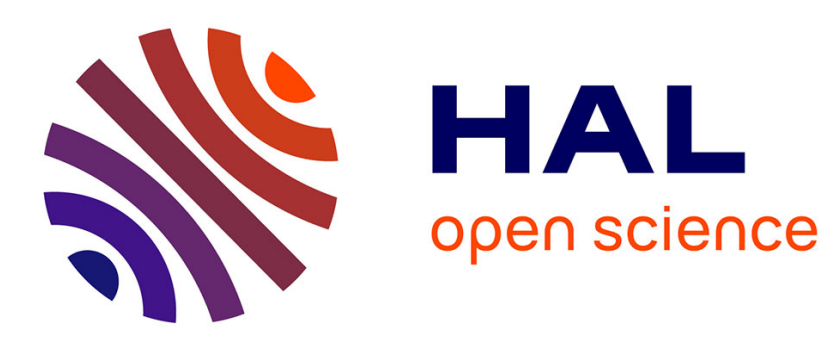

\title{
Comparaison des cinétiques de réaction de la résine RTM6 à l'aide des diagrammes TTT
}

\author{
Florentin Berthet
}

\section{To cite this version:}

Florentin Berthet. Comparaison des cinétiques de réaction de la résine RTM6 à l'aide des diagrammes TTT. Revue des composites et des matériaux avancés = Journal of Composite and Advanced Materials, 2010, 20 (1), p.25-40. 10.3166/RCMA.20.25-40 . hal-01851417

\section{HAL Id: hal-01851417 https://hal.science/hal-01851417}

Submitted on 6 Nov 2019

HAL is a multi-disciplinary open access archive for the deposit and dissemination of scientific research documents, whether they are published or not. The documents may come from teaching and research institutions in France or abroad, or from public or private research centers.
L'archive ouverte pluridisciplinaire HAL, est destinée au dépôt et à la diffusion de documents scientifiques de niveau recherche, publiés ou non, émanant des établissements d'enseignement et de recherche français ou étrangers, des laboratoires publics ou privés. 


\title{
Comparaison des cinétiques de réaction de la résine RTM6 à l'aide des diagrammes TTT
}

\author{
Florentin Berthet
}

Université de Toulouse, INSA, UPS, Mines Albi, ISAE, ICA (Institut Clément Ader)

Ecole des Mines d'Albi-Carmaux, CROMeP

Campus Jarlard, Route de teillet

F-81013 Albi CT cedex 09

Florentin.berthet@enstimac.fr

RÉSUMÉ. L'objet de cet article est de proposer un diagramme temps température transformation (TTT) de la résine RTM6 sur la base de résultats publiés. La résine RTM6 est une résine utilisée en aéronautique. Le diagramme TTT donne une représentation graphique de l'évolution de la cuisson isotherme et des zones de transition (gel, vitrification). La variation de la cinétique de cuisson de part et d'autre de la vitrification est également représentée. Au travers des différents jeux de paramètres existants l'objet est également de représenter la dispersion que l'on peut avoir sur ces cinétiques.

ABSTRACT. The purpose of this article is to propose a time temperature transformation diagram) (T.T.T diagram) of the resin RTM6 on the basis of published datas. RTM6 resin is a resin used in aeronautics. The TTT diagram gives a graphical representation of the evolution of isothermal curing and zones of transition (gel, vitrification). Variation of the kinetics of curing on both sides the vitrification line is also illustrated. Through existing sets of parameters, the object is also to represent the dispersal which we can have on these kinetics MOTS-CLÉS : diagramme TTT, RTM6, résine, liquide, vitrification, cinétique de cuisson. KEYWORDS: TTT Diagram, RTM6, liquid, resin, vitrification, cure kinetic. 


\section{Introduction}

La résine RTM6 est une résine polyepoxyde monocomposant classe $180{ }^{\circ} \mathrm{C}$ largement employée industriellement dans le secteur aéronautique. Cette résine est mise en œuvre par les procédés de fabrication de composites par voie liquide notamment par le procédé d'infusion de résine par le vide (LRI liquid resin infusion) ou par injection sur renfort ou RTM (resin transfer molding).

Ces procédés se décomposent en deux phases. La résine est injectée (ou aspirée) au travers d'un tissu sec, c'est la phase d'imprégnation. Dans un deuxième temps la résine est cuite par l'application d'un cycle de température. La connaissance de la cinétique de cuisson d'une résine est importante pour pouvoir ajuster les cycles de cuisson du composite à la pièce à réaliser.

\section{Présentation du diagramme TTT}

Le diagramme TTT (temps, température transformation) représente sur un graphique en deux dimensions, les courbes d'iso conversion (avancement de la résine) avec le temps en abscisse, et la température en ordonnée.

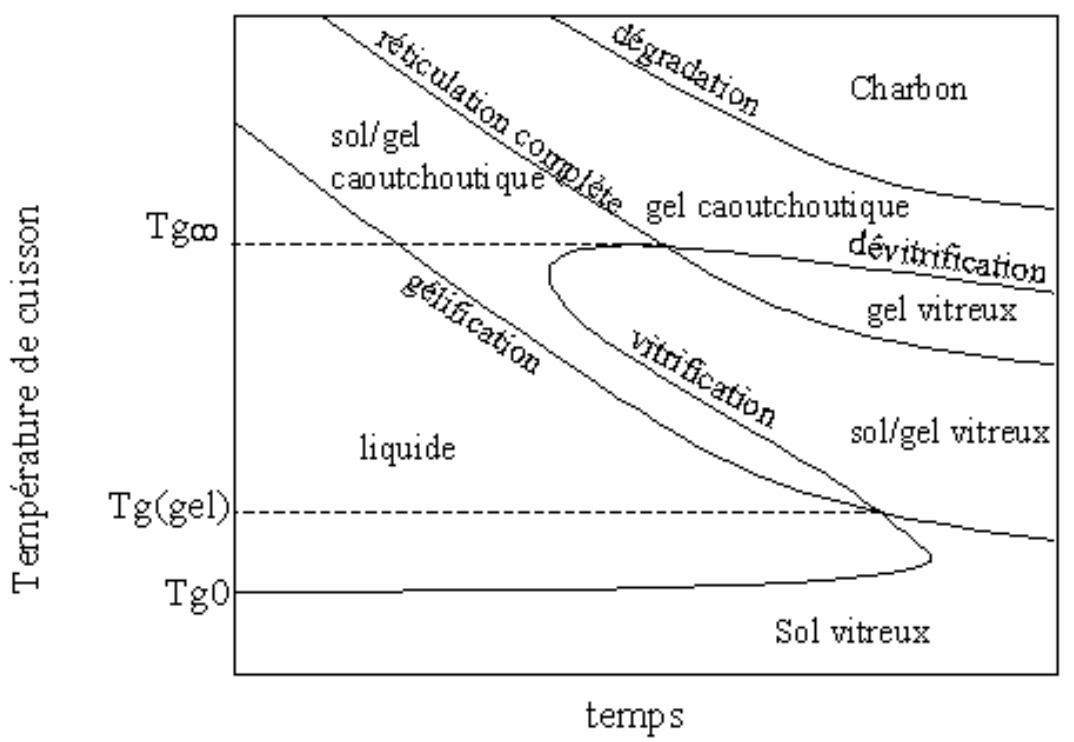

Figure 1. Diagramme TTT d'un thermodurcissable (Barrère et al., 1997) 
Le diagramme TTT montre également les températures critiques et les différents états du polymère. On se reportera utilement à l'article de (Barrère et al., 1997) évoquant le diagramme TTT.

- $\operatorname{Tg} 0$ : température de transition vitreuse initiale

- Tgo: température de transition vitreuse du thermodurcissable totalement réticulé

$-\operatorname{Tg}(\mathrm{gel}): \operatorname{Tg}$ quand vitrification et gel coincident.

La courbe de gélification marque l'apparition d'un réseau macromoléculaire infini. Cette courbe correspond à un degré de conversion bien défini, agel. Le passage de la gélification est sans incidence sur la cinétique de réticulation, par contre après le passage de la gélification la viscosité du thermodurcissable est telle qu'il ne peut plus être mis en forme. Le passage du gel est détecté au moyen d'une mesure de viscosité en fonction du temps.

Une courbe importante représentée sur le diagramme TTT est la courbe de vitrification. A gauche de la vitrification les courbes d'isoconversion sont valables. A droite de la courbe de vitrification, la réaction suit une cinétique différente. Deux cas de figure peuvent se présenter, soit la cinétique utilisée prend en compte le ralentissement de cinétique provoqué par le passage de la vitrification (modèle de diffusion), soit elle ne le prend pas en compte. Dans le cas où le ralentissement est pris en compte les courbes d'isoconversion sont également vraies à droite de la courbe de vitrification, ce cas de figure se remarque car les courbes d'isoconversion sont alors presque parallèles à l'axe des abscisses. La cinétique de réaction est très ralentie par le passage de la vitrification. La courbe de vitrification marque le moment où la température de transition vitreuse $\mathrm{Tg}$ de la résine égale la température de cuisson.

L'objectif de cet article est de donner le diagramme TTT de la résine RTM6 afin de l'utiliser pour la cuisson.

\section{Position du problème}

Considérons le degré de conversion $\alpha$ (également appelé avancement) comme défini par l'équation [1].

$$
\alpha=\frac{\int_{T_{o}}^{T_{f}} \frac{d H}{d t} d T}{\Delta H_{T}}
$$

Cette équation [1] exprime la fraction de chaleur dégagée par la réaction par rapport à la chaleur totale dégagée par la réaction. $\alpha$ évolue donc entre 0 et 1 . 
La vitesse de réaction s'exprime :

$$
\begin{aligned}
& \frac{d \alpha}{d t}=\left(k_{1}+k_{2} \alpha^{m}\right)(1-\alpha)^{n} \\
& k_{1}=A_{1} * \exp \left(-\frac{E_{1}}{R T}\right) \\
& k_{2}=A_{2} * \exp \left(-\frac{E_{2}}{R T}\right)
\end{aligned}
$$

Remarque $\quad \ln \left(k_{1}\right)=\ln \left(A_{1}\right)-\frac{E_{1}}{R T}$

Donc l'énergie d'activation est reliée linéairement au ln du facteur préexponentiel A.

$-\frac{d \alpha}{d t}:$ la vitesse de réaction

- $\mathrm{k} 1, \mathrm{k} 2$ : les coefficients de la vitesse de réaction suivant une loi d'Arrhenius.

$-\mathrm{m}, \mathrm{n}$ : les ordres de réaction

$-\mathrm{E}_{1}, \mathrm{E} 2(\mathrm{~J} / \mathrm{mol})$ : les énergies d'activation

$-A_{1}, A_{2}($ min-1) : les coefficients préexponentiels

$-\mathrm{R}(\mathrm{J} /(\mathrm{mol} * \mathrm{~K}))$ : la constante 8.314

L'équation [2] est bien connue comme « loi de Kamal Sourour ».

Les coefficients de cette équation peuvent être déterminés par différentes méthodes (Ryan et al., 1979 ; Karkanas et al., 2000).

Dans la littérature on trouve plusieurs déterminations de ces coefficients pour la RTM6.

\begin{tabular}{|l|c|c|c|c|c|c|}
\hline & $\mathrm{E} 1(\mathrm{~J} / \mathrm{mol})$ & A1 (min-1) & E2 (J/mol) & A2 (min-1) & $\mathrm{m}$ & $\mathrm{n}$ \\
\hline Karkanas et al., 1996 & 70200 & $1,22 \mathrm{E}+06$ & 53200 & $3,99 \mathrm{E}+05$ & 1,28 & 1,51 \\
\hline Karkanas et al., 2000 & 74690 & $4,50 \mathrm{E}+06$ & 58370 & $1,30 \mathrm{E}+06$ & 1,216 & 1,284 \\
\hline $\begin{array}{l}\text { Navabpour } \text { et al., } \\
2006\end{array}$ & 74000 & $1,66 \mathrm{E}+06$ & 54490 & $4,44 \mathrm{E}+05$ & 1,14 & 1,38 \\
\hline
\end{tabular}

Tableau 1. Valeurs des coefficients selon différents auteurs 
Le calcul de ces paramètres de la cinétique de réaction passe par la réalisation de DSC isothermes à plusieurs températures et par l'optimisation des paramètres qui reproduisent au mieux les données expérimentales.

Après la vitrification, la cinétique de la réaction est physiquement liée à la diffusion. Pour prendre en compte la diffusion on modifie les coefficients $\mathrm{k}$ de la façon suivante :

$$
\frac{1}{k_{i v}}=\frac{1}{k_{i}}+\frac{1}{k_{d}}
$$

$k_{i v}$ : vrai coefficient du terme i

$$
k_{d}=A_{d} * \exp \left(-\frac{E_{d}}{R T}\right) \exp \left(-\frac{b}{f}\right)
$$

$$
f=0.00048\left(T-T_{g}\right)+0.025
$$

On obtient donc en remplaçant $k$ par le coefficient vrai $\mathrm{k}_{\mathrm{iv}}$ dans [2] une équation de la forme :

$$
\frac{d \alpha}{d t}=\left(\frac{k_{1}^{*} k_{d}}{k_{1}+k_{d}}+\frac{k_{2} * k_{d}}{k_{2}+k_{d}} \alpha^{m}\right)(1-\alpha)^{n}
$$

Les coefficients de diffusion sont rassemblés dans le tableau 2.

\begin{tabular}{|l|c|c|c|}
\hline & Ed (J/mol) & $\operatorname{Ad}(\min -1)$ & $\mathrm{b}$ \\
\hline Karkanas et al., 1996 & 136800 & $3.84 * 10^{20}$ & 0.243 \\
\hline
\end{tabular}

Tableau 2. Valeurs des coefficients de diffusion selon Karkanas et al., (1996)

$$
T_{g}=T_{g 0}+\frac{\left(T_{g \infty}-T_{g o}\right) \lambda \alpha}{1-(1-\lambda) \alpha}
$$

L'équation [10] est connue sous le nom d'équation de Di Benedetto, elle relie la $\mathrm{Tg}$ à l'avancement de la réaction au moyen du coefficient $\lambda$. Cette équation permet de tracer la courbe de vitrification connaissant le paramètre $\lambda$. 
Dans notre cas, pour la résine RTM6 $\lambda=0.435$ (Karkanas et al., 2000 II)

\begin{tabular}{|l|c|c|}
\hline & $\mathrm{Tg} 0$ & $\mathrm{Tg} \infty$ \\
\hline Karkanas et al., 2000 II & $-11^{\circ} \mathrm{C} 0$ & $206^{\circ} \mathrm{C}$ \\
\hline
\end{tabular}

Tableau 3.Valeurs des températures critiques (Karkanas et al., 2000 II)

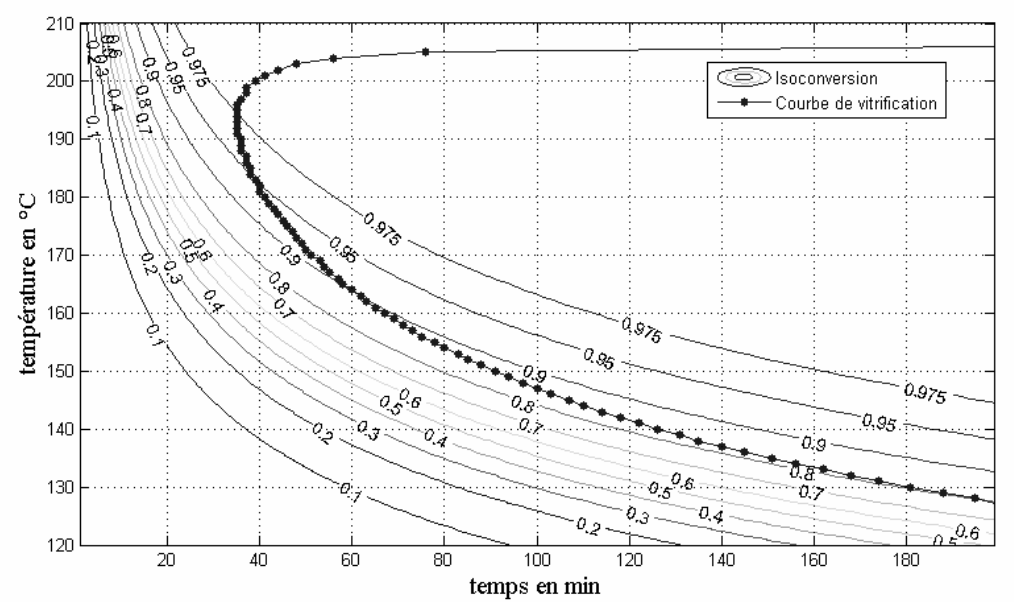

Figure 2. Diagramme TTT de la résine RTM6 selon les données de Karkanas et al., 1996, sans prendre en compte la diffusion

La figure 2 correspond à l'utilisation de la formule [2]. On remarque sur la figure 2 que les courbes d'isoconversion conservent une pente importante même à droite de la courbe de vitrification. Sur cette figure, les courbes d'isoconversion ne sont plus correctes à droite de la courbe de vitrification. En première approximation la conversion est figée au passage de la vitrification. Réellement la vitesse est très ralentie mais non nulle.

Les courbes d'isoconversion présentent l'allure typique de décroissance du temps avec l'augmentation de la température.

Sur la figure 3, qui correspond à la formule [9], la diffusion a été prise en compte, en conséquence les courbes d'isoconversion sont valables de part et d'autre de la courbe de vitrification. Sur cette figure on observe que la pente des courbes d'isoconversion est faible à droite de la courbe de vitrification. Cela reproduit le fait que la cinétique est nettement ralentie après le passage de la vitrification. En comparant les figures 2 et 3 on voit qu'à gauche de la vitrification les courbes d'isoavancement sont équivalentes, à droite de la courbe de vitrification on observe 
une nette différence entre les figures. La prise en compte de la diffusion sur la figure 3 a un effet net.

Nous avons trouvé une valeur de $\alpha$ gel de 0.59 qui est une valeur qui tombe dans la plage ordinairement admise de 0.55 à 0.8 (Barrère et al., 1997).

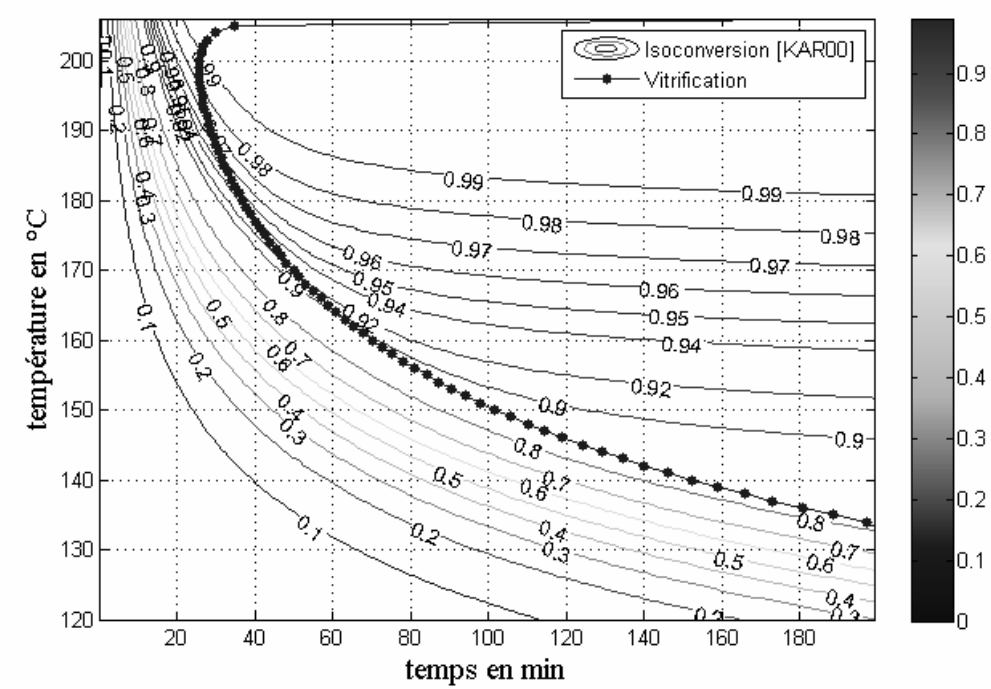

Figure 3. Diagramme TTT de la résine RTM6 selon les données de Karkanas et al., 1996, en prenant en compte la diffusion

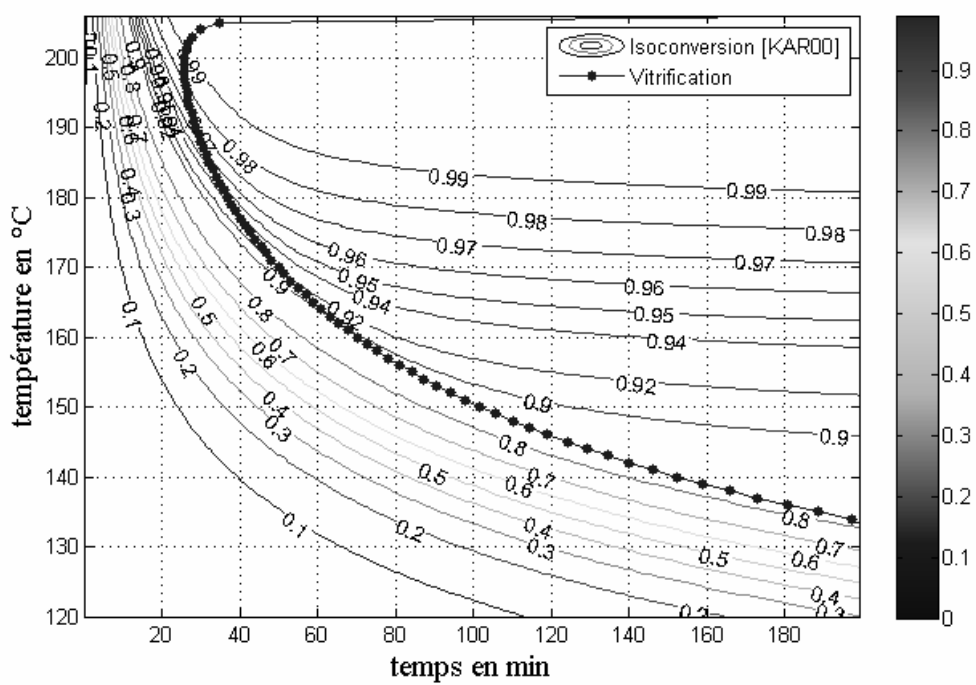

Figure 4. Diagramme TTT de la résine RTM6 selon les données de Karkanas et al., 2000 , en prenant en compte la diffusion 
Ce qui donne une température $\mathrm{Tg}$ gel de $72{ }^{\circ} \mathrm{C}$. A rapprocher de l'article de I. Elsawi (2009) qui disait qu'en dessous de $90^{\circ} \mathrm{C}$ la réaction pouvait être négligée.

Ce qui signifie qu'en dessous de cette température la vitrification se produit avant la gélification, qui compte tenu du fort ralentissement de la réaction empêche la gélification.

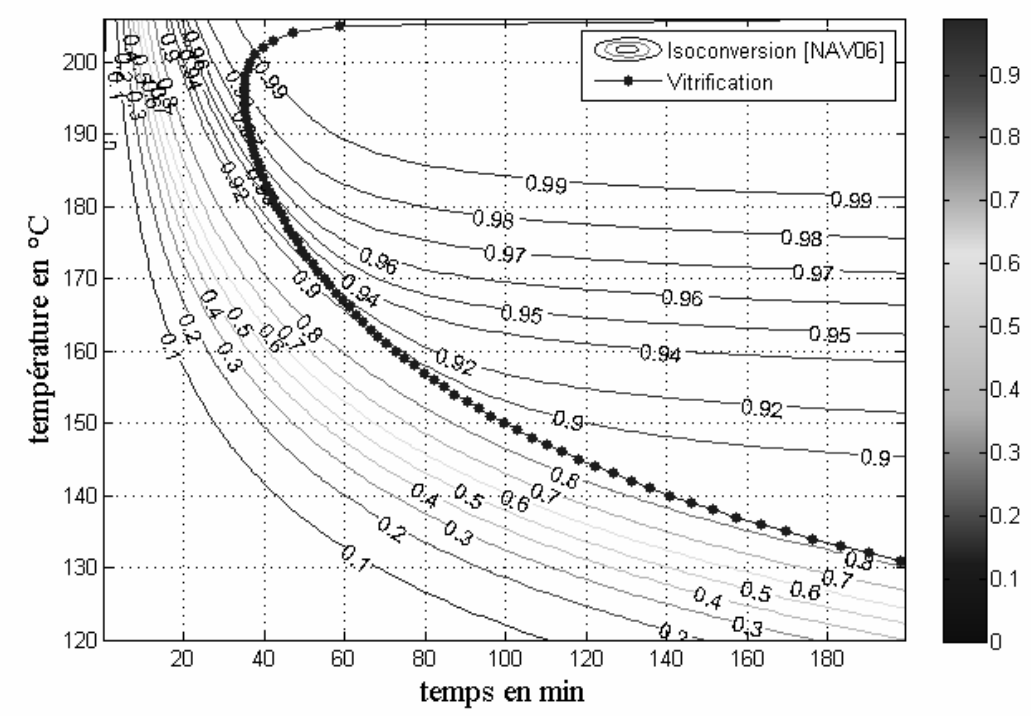

Figure 5. Diagramme TTT de la résine RTM6 selon les données de Navabpour et al., 2006, en prenant en compte la diffusion

Si l'on superpose les diagrammes TTT que l'on obtient pour la résine RTM6 selon trois sources différentes on obtient la figure 6 .

Sur la figure 6 on observe que le tracé correspondant aux coefficients de Navabpour présente un retard cinétique par rapport au tracé correspondant au coefficient de Karkanas et Partridge. Cela apparaît au travers du décalage des courbes d'isoconversion et de vitrification. Ces différences de cinétiques observées peuvent traduire différentes réalités :

- la différence de cinétique entre des lots différents ;

- la différence de cinétique découlant de la modélisation et de l'ajustement des paramètres ;

- le fait que les jeux de paramètres décrivant la cinétique ne sont pas uniques. 


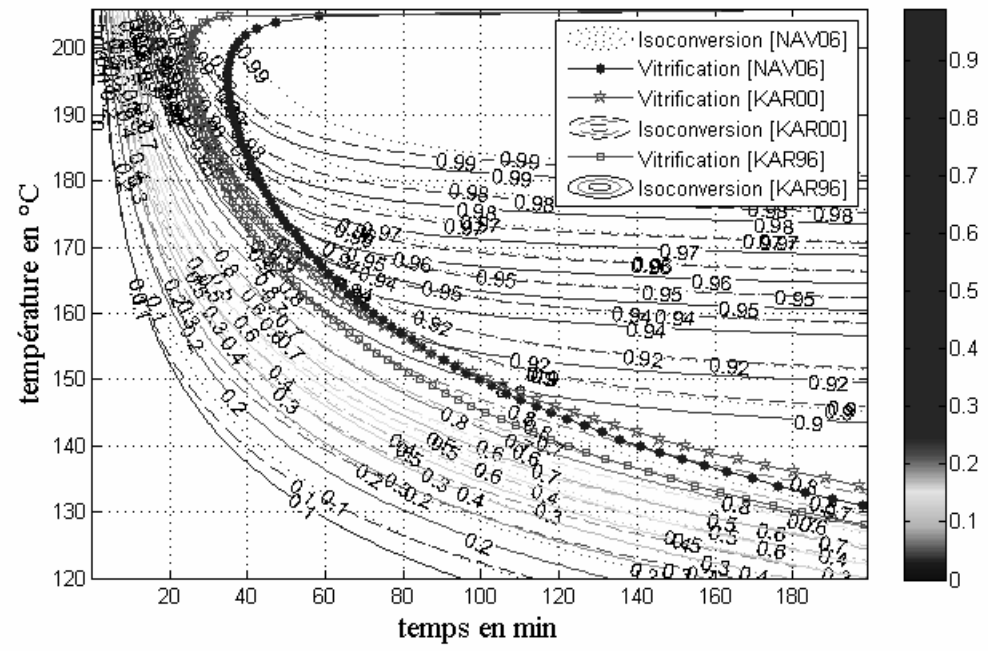

Figure 6. Comparaison des diagrammes TTT obtenus avec les coefficient de trois sources différentes (Karkanas et al., 1996 ; 2000 ; Navabpour et al. 2006)

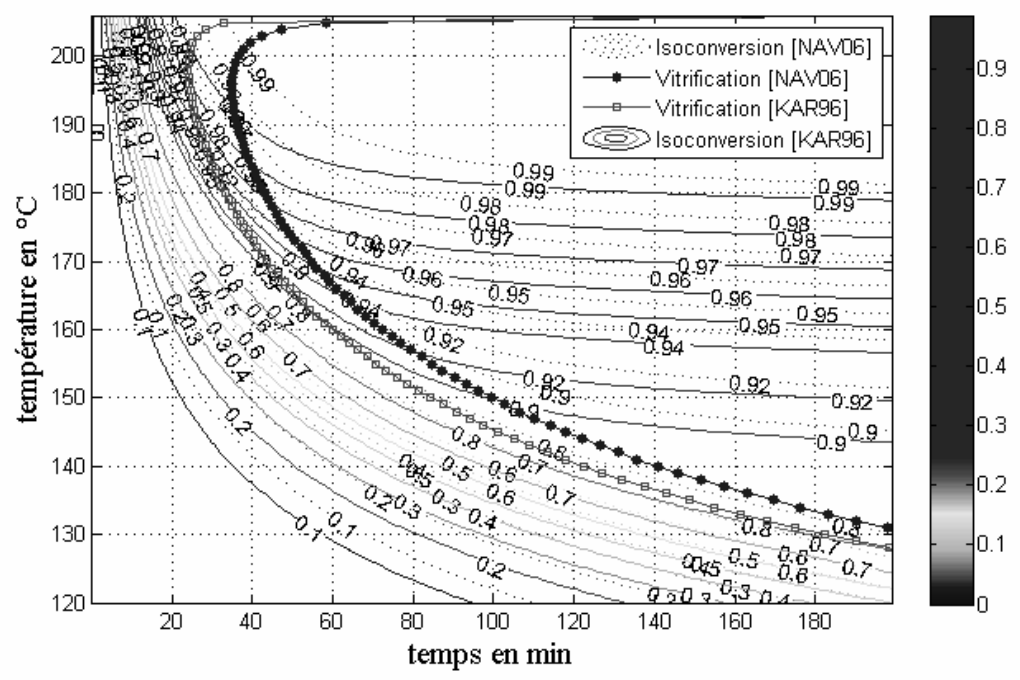

Figure 7. Comparaison des diagrammes TTT obtenus avec les coefficients de deux sources différentes (Karkanas et al., 1996 ; Navabpour et al., 2006) 
De plus, la détermination des paramètres est relativement sensible aux données expérimentales ce qui fait que la précision du graphe TTT est relative (figures 7 et 8). La figure 7 montre le décalage entre les jeux de paramètres extrêmes.

Les paramètres $(\lambda, m, n)$ sont considérés comme constants dans le modèle utilisé, en fait ils présentent une dépendance à la température. Mais cela rendrait les choses plus complexes et nous y perdrions en compréhension.

On remarque sur la figure 7 que les courbes de vitrification sont séparées d'une quinzaine de minute (ce qui est important) à $180^{\circ} \mathrm{C}$.

\section{Validation}

Les diagrammes TTT ayant été tracés il est intéressant des les comparer à d'autres résultats.

\section{Validation bibliographique documentation fournisseur}

La documentation du fabriquant de la RTM6 donne des informations sur l'avancement obtenu avec différents cycles de cuisson.

75 minutes à $160^{\circ} \mathrm{C}$ donne un avancement de $91 \%$, ce qui correspond avec la figure 5 .

75 minutes à $160^{\circ} \mathrm{C}$ et post cuisson de 120 minutes à $180{ }^{\circ} \mathrm{C}$, donne un avancement de $94 \%$. La figure 5 prédit dans ce cas $99 \%$ d'avancement ce qui est surestimé (5\%). Mais nous reviendrons sur ce point un peu plus loin.

120 minutes à $160^{\circ} \mathrm{C}$ donne un avancement de $91 \%$. La figure 5 conduit à un avancement de $94 \%$ (surestimation $3 \%$ ).

60 minutes à $180^{\circ} \mathrm{C}$ donne un avancement de $95 \%$. La figure 5 conduit à un avancement de $98 \%$ (surestimation $3 \%$ ).

90 minutes à $180^{\circ} \mathrm{C}$ donne un avancement de $96 \%$. La figure 5 conduit à un avancement de $985 \%$ (surestimation $2.5 \%$ ).

120 minutes à $180{ }^{\circ} \mathrm{C}$ donne un avancement de $96 \%$. La figure 5 conduit à un avancement de $99 \%$ (surestimation $3 \%$ ).

Nous pouvons donc constater que sur ce tableau 4 les diagrammes TTT proposés surestiment de quelques \% l'avancement constaté. La comparaison à la fiche technique du produit nous porte à penser que le diagramme TTT le plus fidèle est celui utilisant les données de Navabpour et al., (2006).

Il apparaît cependant que les trois jeux de paramètres sont valables (Karkanas et al., 1996 ; 2000) et (Navabpour et al., 2006). 


\begin{tabular}{|c|c|c|c|c|}
\hline & $\begin{array}{c}\text { Hexcel, } \\
2007\end{array}$ & $\begin{array}{c}\text { Karkanas et } \\
\text { al., } 1996\end{array}$ & $\begin{array}{c}\text { Karkanas et } \\
\text { al., } 2000\end{array}$ & $\begin{array}{l}\text { Navabpour } \\
\text { et al., } 2006\end{array}$ \\
\hline 75 minutes à $160^{\circ} \mathrm{C}$ & 91 & 93 & 91 & 91 \\
\hline $\begin{array}{l}75 \text { minutes à } 160^{\circ} \mathrm{C} \\
\text { et post-cuisson de } 120 \text { minutes à } 180^{\circ} \mathrm{C}\end{array}$ & 94 & & & \\
\hline 120 minutes à $160^{\circ} \mathrm{C}$ & 91 & 94.5 & 94 & 93 \\
\hline 60 minutes à $180^{\circ} \mathrm{C}$ & 95 & 98 & 98 & 97 \\
\hline 90 minutes à $180^{\circ} \mathrm{C}$ & 96 & 98.5 & 98.5 & 98 \\
\hline 120 minutes à $180^{\circ} \mathrm{C}$ & 96 & 99 & 99 & 98 \\
\hline
\end{tabular}

Tableau 4. Comparaison des avancements en \% selon différents cycles de cuisson et selon différents auteurs

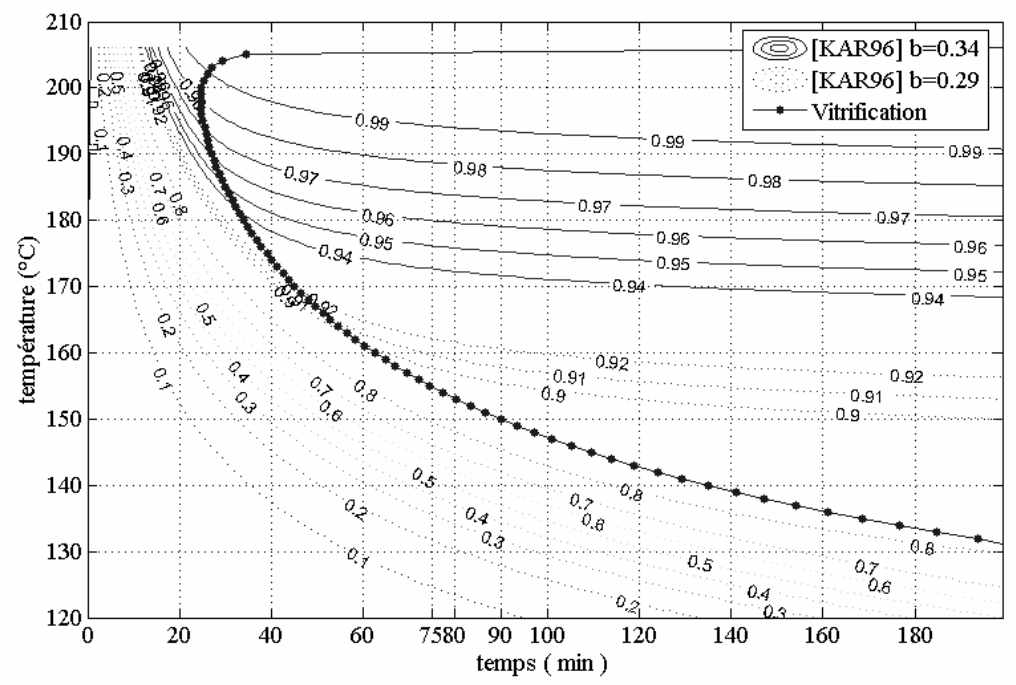

Figure 8. Diagramme TTT avec $b=0.29$ pour $T=160$ et $b=0.34$ pour $T=180^{\circ} \mathrm{C}$ (Karkanas et al., 1996)

La figure 8 illustre l'utilisation de deux coefficients de diffusion b différents $\mathrm{b}=0.29$ pour les niveaux de conversion inférieurs à 0.93 (température de $160^{\circ} \mathrm{C}$ ) et $\mathrm{b}=0.34$ pour les avancements supérieurs à 0.93 (pour la température de $180^{\circ} \mathrm{C}$ ).

\section{Amélioration}

Une alternative pour prendre en compte la diffusion dérive de l'approche de Ruiz (Ruiz et al., 2008). 


$$
f(\alpha)=\frac{1}{1+\exp \left[C_{F}\left(\alpha-\alpha_{c}(T)\right)\right]}
$$

avec $f$ intervenant en facteur multiplicatif de la loi de Kamal sourour, si bien que [2] devient

$$
\frac{d \alpha}{d t}=f(\alpha) *\left(k_{1}+k_{2} \alpha^{m}\right)(1-\alpha)^{n}
$$

La différence avec ce que proposait Ruiz consiste à prendre $\alpha_{c}(T)$ non pas constant mais dépendant de $\mathrm{T}$ (en fait la valeur au passage de la vitrification).

$\mathrm{C}_{\mathrm{F}}=300$ (valeur adaptée pour bien reproduire les données du fabriquant)

De même on prend

$$
\begin{aligned}
& \mathrm{m}=1.52-1.94 \mathrm{e}-3 *\left(\mathrm{~T}\left({ }^{\circ} \mathrm{C}\right)\right) \\
& \mathrm{n}=1.90-3 . \mathrm{e}-3 *\left(\mathrm{~T}\left({ }^{\circ} \mathrm{C}\right)\right)
\end{aligned}
$$

comme dans (Karkanas et al., 1996) et on obtient la figure 9.

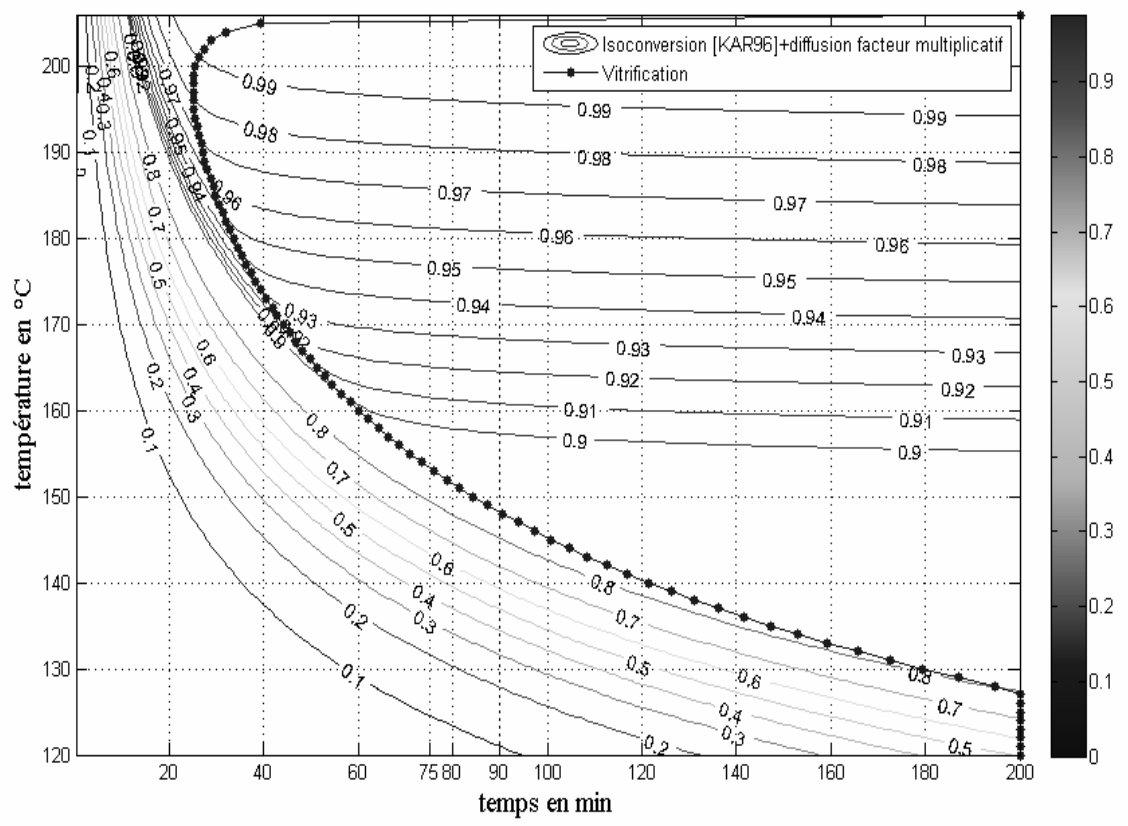

Figure 9. Diagramme TTT diffusion prise en compte par facteur multiplicatif 


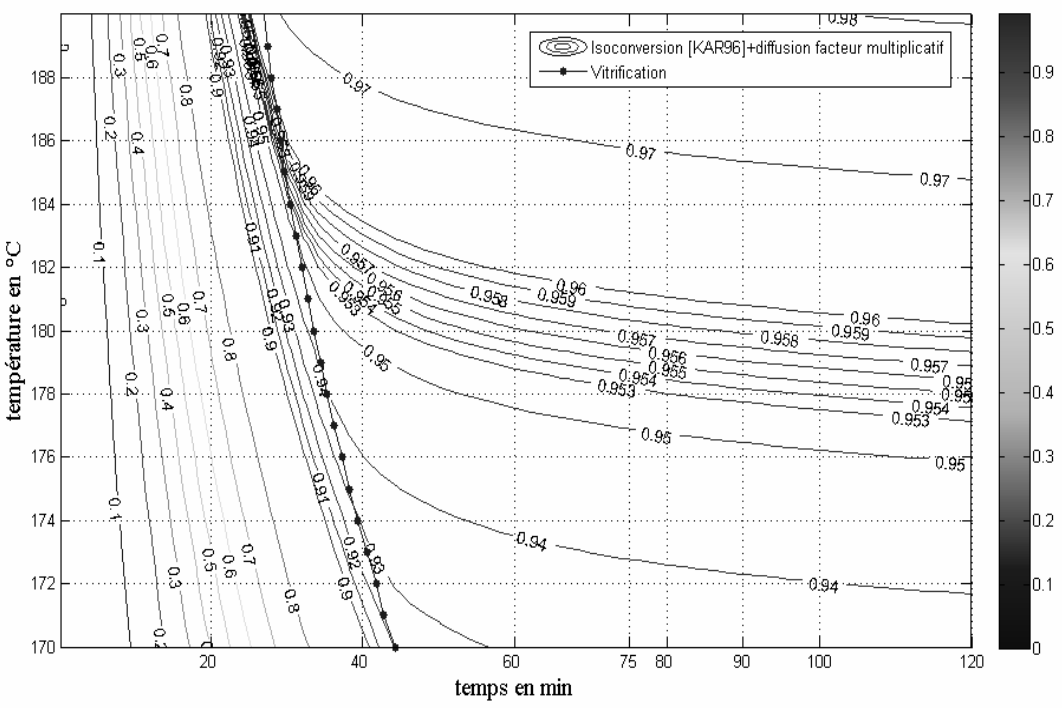

Figure 10. Diagramme TTT diffusion prise en compte par facteur multiplicatif (Zoom sur $180^{\circ} \mathrm{C}$ )

Le tableau 5 donne la synthèse des avancements prédits sur la base de la figure 9 .

\begin{tabular}{|c|c|c|}
\hline & $\begin{array}{c}\text { Hexcel, } \\
2007\end{array}$ & figure 9 \\
\hline 75 minutes à $160^{\circ} \mathrm{C}$ & 91 & 90.3 \\
\hline $\begin{array}{l}75 \text { minutes à } 160^{\circ} \mathrm{C} \\
\text { et post cuisson de } 120 \text { minutes à } 180^{\circ} \mathrm{C}\end{array}$ & 94 & 96 \\
\hline 120 minutes à $160^{\circ} \mathrm{C}$ & 91 & 91 \\
\hline 60 minutes à $180^{\circ} \mathrm{C}$ & 95 & 95.6 \\
\hline 90 minutes à $180^{\circ} \mathrm{C}$ & 96 & 95.8 \\
\hline 120 minutes à $180^{\circ} \mathrm{C}$ & 96 & 96 \\
\hline
\end{tabular}

Tableau 5. Comparaison des avancements en \% selon différents cycles de cuisson et selon différents auteurs

Revenons sur le cycle 75 minutes à $160^{\circ} \mathrm{C}$ suivi d'une post-cuisson de 120 minutes à $180^{\circ} \mathrm{C}$. Selon la figure 9,75 minutes à $160^{\circ} \mathrm{C}$ donnent un avancement de 0.903 . 


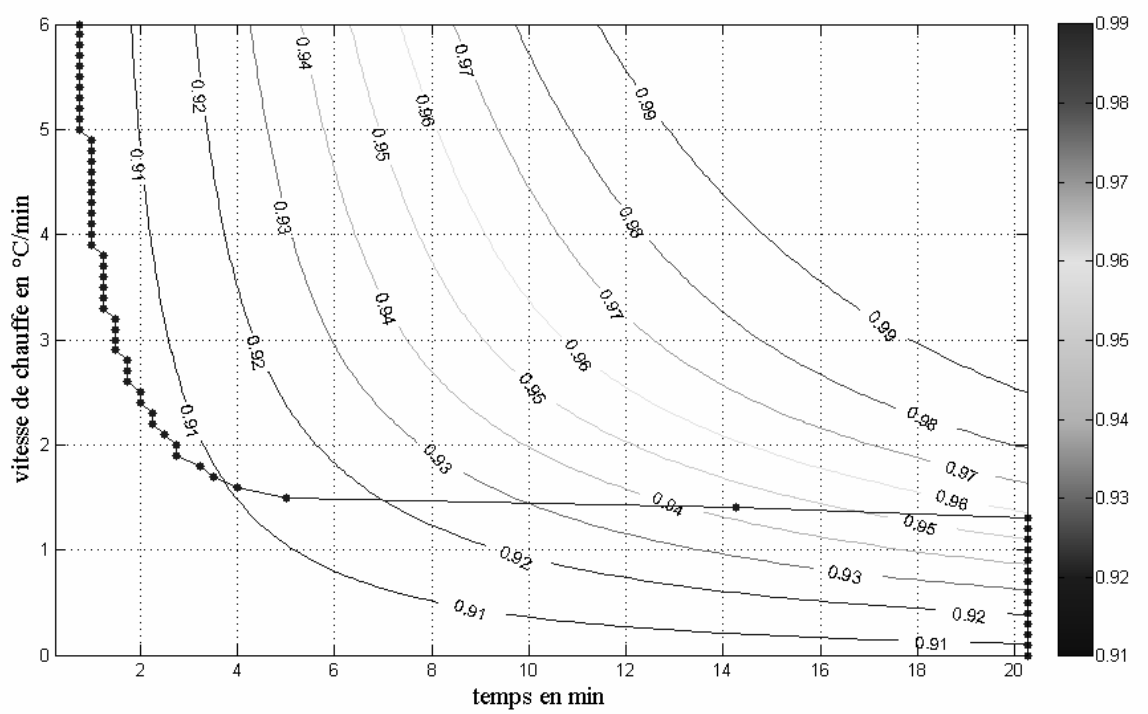

Figure 11. Diagramme d'isoconversion en vitesse de chauffe avec avancement initial de 0.903 (valeur issue du tableau 5)

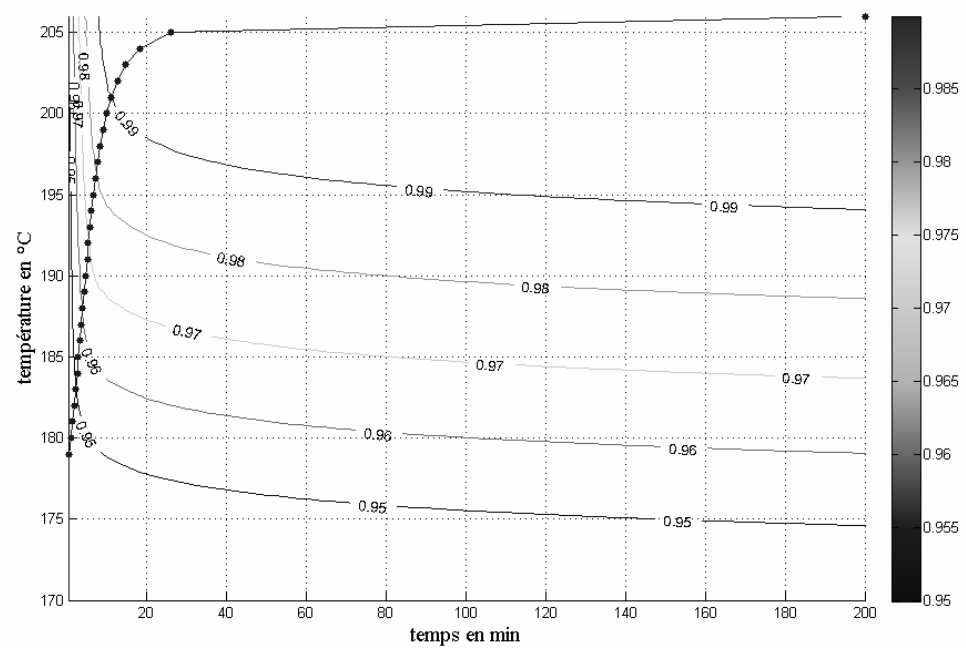

Figure 12. Diagramme d'isoconversion en isotherme avec avancement initial de 0.94 
Selon la figure 11 en chauffant de 160 à $180^{\circ} \mathrm{C}$ en 20 minutes (à $1{ }^{\circ} \mathrm{C}$ par minute) on ne dépasse pas la $\mathrm{Tg}$ du matériau et après 20 minutes on a 0.94 d'avancement.

Sur la figure 11 la courbe avec des carrés traduit le passage de la température de cuisson au-dessus de la $\mathrm{Tg}$ de la résine. A $1{ }^{\circ} \mathrm{C} / \mathrm{min}$ on reste en dessous de la $\mathrm{Tg}$ du matériau. Par contre à $2^{\circ} \mathrm{C} / \mathrm{min}$ la température de cuisson passe au-dessus de la $\mathrm{Tg}$ du matériau.

Selon la figure 12 on voit qu'au terme de la post-cuisson de 120 minutes à $180^{\circ} \mathrm{C}$ on a un avancement calculé de $96 \%$ à comparer aux $94 \%$ annoncés sur la fiche technique du fabriquant.

\section{Conclusion}

Au regard de la comparaison des diagrammes TTT obtenus avec trois jeux de paramètres issus de la bibliographie on peut tout d'abord remarquer la similitude d'allure des diagrammes obtenus. Néanmoins des différences importantes existent, notamment au niveau du temps entre les diagrammes TTT obtenus. Cela traduit la réalité de dispersion au niveau des vitesses de réaction, ainsi que la dispersion dans la détermination des paramètres cinétiques.

Néanmoins ces graphiques permettent d'appréhender le comportement de la résine et sont une aide à la prédiction de l'avancement obtenu par un cycle de cuisson.

Ces graphiques mettent en avant le rôle particulier de la vitrification par rapport à l'avancement et à la cinétique de réaction de la résine, les cinétiques étant nettement différentes de part et d'autre de la vitrification.

\section{Bibliographie}

Barrère C., dal Maso F., «résines époxy reticulées par les polyamines : structures et propriétés, Revue de l'institut français du pétrole, vol. 52, n 3, mai-juin, 1997, p. 317-335.

Elsawi I., Olivier P., Demont P., Laurent C., Peigney A., «Comportement rhéologique et cinétique d'une résine époxyde chargée de nanotubes de carbone double parois », Journées nationales des composites, JNC16, 2009, Toulouse.

HEXCEL, HexFlow RTM6, $180^{\circ} \mathrm{C}$ epoxy system for resin transfer moulding monocomponent system, Product Data, March 2007.

Karkanas P.I., Partridge I.K., "Modelling the cure of a commercial Epoxy resin for applications in resin transfer molding", Polymer International, 1996, vol. 41, p. 183-191.

Karkanas P.I., Partridge I.K., "Cure modelling and monitoring of epoxy/amine resin Systems I. Cure Modeling”, Journal of applied Polymer Science, 2000, vol. 77, p. 1419-1431. 
Karkanas P.I., Partridge I.K., "Cure modelling and monitoring of epoxy/amine resin Systems II. Network formation and chemoviscosity modelling", Journal of applied Polymer Science, 2000, vol. 77, p. 2178-2188.

Navabpour P., Nesbitt A., Degamber B., Fernando G., Mann T., R. Day, “Comparison of the curing kinetics of the RTM6 epoxy Resin System Using differential scanning calorimetry and a microweave-heated calorimeter", Journal of applied Polymer Science, 2006, vol. 99 , p. 3658-3668.

Ruiz E., Billotte C., "Predicting the cure of thermosetting Polymers", Polymer composites, 2008, p. 1-8.

Ryan M.E., Dutta A., "Kinetics of epoxy cure: a rapid technique for kinetic parameter estimation", Polymer, 1979, vol. 20, February, p. 203-206. 